\title{
Avaliação da persistência na lactação da raça Guzerá, utilizando modelos de regressão aleatória
}

[Evaluation of lactation persistency of Guzerat cows using random regression models]

\author{
L.S. Freitas ${ }^{1}$, M.A. Silva ${ }^{2 *}$, R.S. Verneque ${ }^{3}$, B.D. Valente ${ }^{1}$, G.S. Corrêa ${ }^{1}$, R.F. Ferreira ${ }^{4}$, \\ M.G.C.D. Peixoto ${ }^{3}$, G.G. Santos ${ }^{1}$ \\ ${ }^{1}$ Aluno de pós-graduação - EV-UFMG - Belo Horizonte, MG \\ ${ }^{2}$ Escola de Veterinária - UFMG \\ Caixa Postal 567 \\ 30123-970 - Belo Horizonte, MG \\ ${ }^{3}$ Embrapa Gado de Leite, Juiz de Fora, MG \\ ${ }^{4}$ Aluna de pós-graduação - UFU - Uberlândia, MG
}

\begin{abstract}
RESUMO
Estimaram-se a herdabilidade e as correlações genéticas e de ambiente permanente entre seis medidas de persistência da lactação de vacas da raça Guzerá, utilizando modelo de regressão aleatória. Foram considerados 8276 registros de produção de leite no dia do controle, na primeira lactação, de 1021 vacas. A regressão aleatória foi calculada pela função logarítmica de Ali e Schaeffer e pelo polinômio de Legendre, obtendo-se os coeficientes para os efeitos fixos, genético aditivo e de ambiente permanente. A função que mais se adequou aos dados foi a de Ali e Schaeffer, mas apresentou problemas de convergência. Os resultados evidenciaram que a persistência é uma característica com herdabilidade de valor moderado e de baixa correlação com o valor genético para produção de leite aos 305 dias, indicando a possibilidade de se obter resposta à seleção para mudança na curva de lactação sem afetar negativamente a produção total de leite na lactação. A medida de persistência que calcula a diferença de produção de leite entre as fases intermediária e inicial da lactação apresentou alta correlação com a produção aos 305 dias.
\end{abstract}

Palavras-chave: bovino de leite, herdabilidade, correlação genética, função logarítmica, polinômio de Legendre

\section{ABSTRACT}

The heritability and the genetic and permanent environment correlations were estimated among six different measures of persistency in the lactation of Guzerat cow, using the Random Regression Model. A total of 8,403 records from 1,034 first lactation cows were evaluated. The Random Regression Model was calculated by the logarithmic function of Ali and Schaeffer and Legendre polynomials to get coefficients for fixed, additive genetic and permanent environment effects. Ali and Schaeffer was the function that better fit to the data, but it had convergence problems. The results showed that persistence is a trait with moderate heritability, and low correlation with genetic value for 305-d milk production which allows to select animals in order to alter the format of the curve of production without affecting the total productivity. The measure of persistence that calculates the difference of milk production between the medium and initial phases was highly correlated with $305-d$ milk production.

Keywords: dairy cattle, heritability, genetic correlation, logarithmic function, Legendre polynomial

Recebido em 8 de agosto de 2009

Aceito em 31 de março de 2010

Autor para correspondência (corresponding author)

E-mail: martinho@vet.ufmg.br 


\section{INTRODUÇÃO}

O uso de animais de raças adaptadas às condições de clima e de manejo em sistemas de produção de leite pode propiciar redução expressiva nos custos de produção e aumento da rentabilidade. A raça Guzerá, introduzida no Brasil no final do século XIX, tem sido uma das principais raças de dupla aptidão explorada no país, pela grande capacidade de crescimento e ganho de peso, potencial para produção de leite e resistência aos ecto e endoparasitas (Winkler e Penna, 1992).

As avaliações genéticas para produção de leite realizadas no país baseiam-se na produção de leite acumulada por um período padrão de 305 dias. Esse cálculo, geralmente é realizado por meio da projeção da curva com base em controles leiteiros mensais. Como alternativa às estimativas feitas para as avaliações genéticas, surgiram metodologias que permitiram a utilização da produção no dia do controle.

Análises baseadas na produção no dia do controle permitem definir de forma precisa os grupos contemporâneos e os efeitos ambientais atuantes em cada um, além de ajustar melhor as curvas de lactação com duração menor do que 305 dias (Swalve, 1995). Segundo Jensen (2001), esse tipo de medida permite avaliações mais frequentes de cada animal, o que diminui o intervalo de gerações por possibilitar a utilização de lactações incompletas, além de aumentar o número de observações do mesmo indivíduo permitindo, assim, obter maior acurácia na estimativa do valor genético. Além disso, ao se projetar uma curva de lactação, vacas que apresentam maior persistência na lactação têm a produção de leite total subestimada, e as com menor taxa de persistência têm produções superestimadas. Isso pode causar problemas nas avaliações genéticas de animais que se destinam a reprodutores (Jamrozik e Schaeffer, 1997).

A persistência na lactação é a capacidade de a vaca manter o nível de produção de leite após atingir o pico de lactação e está relacionada à redução dos custos de produção. Como há indicativos da variabilidade genética para esta característica, a seleção, tendo como base a persistência na lactação, pode trazer ganhos econômicos pela redução de custos e pela produção adicional de leite (Jamrozik et al., 1997).

Mais recentemente, a utilização de modelos de regressão aleatória, tornou-se uma alternativa para avaliação de dados longitudinais. Estes predizem coeficientes de regressão que representam o valor genético de cada animal em função do tempo, diferenciando, portanto, dos demais modelos que predizem o valor genético para período determinado. Ao se utilizarem modelos de regressão aleatória para avaliar a curva de lactação com observações no dia do controle, permite-se a formação de uma curva de valores genéticos para produção de leite para cada animal. Isso permitirá selecionar animais com curvas de lactação mais persistentes (Jamrozik e Schaeffer, 1997).

Nas análises mediante uso dos modelos de regressão aleatória não há necessidade do número mínimo de observações por animal. Estas análises permitem estimar componentes de covariância entre controles diferentes, incluindo os períodos em que estes não foram tomados, tornando desnecessários os ajustes na produção de leite (Jamrozik e Schaeffer, 1997; Meyer e Hill, 1997; Schaeffer, 2004).

Os objetivos deste trabalho foram estudar a viabilidade do uso da persistência na lactação como característica a ser incluída nas avaliações genéticas para a raça Guzerá e determinar parâmetros genéticos para esta característica por meio de modelos de regressão aleatória.

\section{MATERIAL E MÉTODOS}

Foram utilizadas informações extraídas da base de dados do Programa Nacional de Melhoramento do Guzerá para Leite, executado pela Embrapa Gado de Leite, em parceria com o Centro Brasileiro de Melhoramento do Guzerá. A base de dados original continha 45026 registros de produção de leite no dia do controle (PLDC), de 3935 vacas puras e mestiças Guzerá. Para maior consistência dos dados, foram selecionadas observações das primeiras lactações de vacas com pais conhecidos e puros. Vacas filhas de touros com menos de três filhas, com menos de cinco controles e mais de 2000 dias de idade foram descartadas. Após a edição dos dados, restaram 8276 observações de 1021 vacas. 
Foram considerados controles de produção de leite entre o sexto e o $305^{\circ}$ dias de lactação no período de 1987 a 2006, intervalados de, no mínimo, 14 dias e, no máximo, 74 dias, visando à obtenção de maior número de informações por animal. Em média, as vacas apresentaram seis controles no período.

A separação dos grupos contemporâneos foi feita de acordo com rebanho-ano e época do controle, sendo esta dividida em duas, abril a setembro e outubro a março, o que permitiu formar 110 classes de efeito fixo classificatório. No modelo de regressão aleatório, foram incluídos os efeitos

$$
Y_{g i j k l}=R A E_{i}+\sum_{m=1}^{n} \beta_{k m} Z_{j l m}+\sum_{m=1}^{n} a_{j m} Z_{j l m}+\sum_{m=1}^{n} p_{j m} Z_{j l m}+e_{g i j k l} \text {, em que }
$$

$Y_{g i j k l}$ é o controle 1 da vaca $j$ no $\left(\mathrm{t}_{\mathrm{j}}\right)$ período de dias em lactação, dentro da classe $i$ (rebanhoano-época do controle), pertencente à subclasse $\mathrm{k}$ (idade-época de parto); $R A E_{i}$ é o efeito fixo de rebanho-ano-época do controle; $e_{i j k l}$ é o efeito aleatório residual associado à $Y_{i j k l} ; \beta_{k m}$ é vetor que contém coeficientes de regressão dos efeitos fixos da produção de leite no dia do controle em função de $t$ e descreve a forma da curva de lactação dentro das classes de idade da vacaépoca de parto; $a_{j m}$ e $p_{j m}$ são vetores contendo coeficientes de regressão aleatórios que descrevem a trajetória do efeito genético aditivo e permanente de ambiente, respectivamente, durante o período de lactação para cada animal; n é o número de coeficientes da função utilizada e $Z_{j l m}$ é um vetor de covariáveis, que representa os respectivos coeficientes das funções matemáticas utilizadas para descrever a curva de lactação. Neste estudo, foram utilizados o polinômio de Legendre e a função logarítmica de Ali e Schaeffer (Ali e Schaeffer, 1987).

O polinômio de Legendre de terceiro grau foi utilizado para descrever as variações aleatórias da curva de lactação em função dos dias de lactação. Esse modelo foi escolhido em razão da grande diferença entre os autovalores das matrizes de covariâncias dos coeficientes de regressão aleatória, além da facilidade computacional, pois a utilização de polinômios com maior grau resultaria em mais coeficientes para serem estimados, o que levaria maior tempo para análise, mas com resultados que apresentariam valores genéticos semelhantes (Cobuci et al., 2006). fixos dos controles agrupados de acordo com cinco classes de idade da vaca - 22 a 36 meses, 37 a 39, 40 a 42, 43 a 47 e 48 a 59 meses -, e em duas épocas de parto, abril a setembro e outubro a março. Esses grupos foram combinados constituindo-se 10 classes de idade-época do parto, pressupondo que cada classe pode apresentar um formato diferente para a curva de lactação.

$\mathrm{O}$ arquivo de genealogia continha 5005 animais.

O seguinte modelo foi utilizado para obtenção dos coeficientes de regressão aleatória:
A conversão das covariáveis de dias em lactação (t) para o polinômio de Legendre é realizada a partir da transformação para uma escala de -1 a 1 (Kirkpatrick et al., 1990), da seguinte forma:

$t=-1+2\left(\frac{\text { Dia do controle }- \text { dia mínimo do controle }}{\text { Dia máximo do controle }- \text { dia mínimo do controle }}\right)$

Os polinômios de Legendre são ortogonalizados (Schaeffer, 2001) e normalizados:

A função de Ali e Schaeffer, utilizada na descrição dos mesmos efeitos, é representada por: $a_{0}+a_{1} c+a_{2} c^{2}+a_{3}\left(\ln \frac{1}{c}\right)+a_{4}\left(\ln \frac{1}{c}\right)^{2}$, em que c equivale a dias em lactação dividido por 305.

Para verificação do modelo de melhor ajuste, foram utilizados a função de verossimilhança (2ln L) e o critério de informação de Akaike (AIC), que é obtido por:

$$
A I C=-2 \ln L+2 p, \text { em que }
$$

-2lnL é o valor da função de verossimilhança encontrado na análise e $\mathrm{p}$ é o número de parâmetros do modelo. Menores valores para 2ln L para o AIC indicam modelos mais adequados aos dados analisados. O critério de informação de Akaike penaliza modelos com maior número de parâmetros.

A persistência na curva de lactação, que é considerada o mais importante componente da 
curva de lactação (Wood, 1967), representa a taxa de declínio da produção de leite após o pico da lactação.

Para cálculo da persistência da lactação e predição dos valores genéticos dos animais, foram utilizadas seis diferentes medidas: $P S_{1}=\left(V g_{280}-V g_{60}\right)$, que indica o diferencial genético para produção de leite entre 60 e 280 dias de produção (Jamrozik et al., 1997); $P S_{2}=\left(\sum_{t=106}^{205} V g_{t}-\sum_{t=6}^{105} V g_{t}\right)$, diferença entre as áreas sob a curva de lactação, nos terços médio e inicial da lactação (Jakobsen et al., 2002); $P S_{3}=\left(\sum_{t=206}^{305} V g_{t}-\sum_{t=6}^{105} V g_{t}\right)$, diferença entre as áreas sob a curva de lactação, nos terços final e inicial da lactação (Jakobsen et al., 2002); $P S_{4}=\sum_{t=61}^{280}\left(V g_{t}-V g_{60}\right), \quad$ somatório das produções de 61 a 280 dias como desvios da produção aos 60 dias de lactação (Jamrozik et al., 1997); $P S_{5}=\sum_{t=60}^{279}\left(V g_{t}-V g_{280}\right)$, somatório da produção de cada dia de 60 a 279 dias, como desvio da produção aos 280 dias (Jakobsen et al., 2002); е $P S_{6}=\left(V g_{290}-V g_{90}\right)$, que indica o diferencial genético para produção de leite entre 90 e 290 dias de lactação (Cobuci et al., 2004).

Deve ser ressaltado que para maiores níveis de persistência na lactação, são desejáveis menores valores para $\mathrm{PS}_{1}, \mathrm{PS}_{2}, \mathrm{PS}_{3}, \mathrm{PS}_{4}$ e $\mathrm{PS}_{6}$, mas, para $\mathrm{PS}_{5}$, são desejados maiores valores.

As variâncias genéticas aditiva e de ambiente permanente foram obtidas por: $\sigma_{a}^{2}=Z_{t}^{\prime} G Z_{t}$ e $\sigma_{e p}^{2}=Z_{t}^{\prime} P Z_{t}$, em que $G$ e $P$ são matrizes de variância e covariância entre os coeficientes de regressão aleatória; $Z_{t}$ é o vetor de covariáveis, obtido a partir das medidas de persistência, para o período $t$ da lactação.

As estimativas dos coeficientes de herdabilidade para as diferentes mensurações da persistência da curva de lactação foram calculadas por:

$$
\hat{\mathrm{h}}_{(\mathrm{n})}^{2}=\frac{\hat{\sigma}_{\mathrm{a}}^{2}(\mathrm{n})}{\hat{\sigma}_{\mathrm{a}}^{2}(\mathrm{n})+\hat{\sigma}_{\mathrm{ep}}^{2}(\mathrm{n})+\left(\mathrm{C}_{\mathrm{n}} * \hat{\sigma}_{\mathrm{e}}^{2}\right)} \text {, em que }
$$

(n= $\left.\mathrm{PS}_{1}, \mathrm{PS}_{2}, \mathrm{PS}_{3}, \mathrm{PS}_{4}, \mathrm{PS}_{5}, \mathrm{PS}_{6}\right)$ e $\mathrm{C}_{\mathrm{n}}$ é constante, dada pelos valores 2, 200, 200, 48620, 48620, 2, para $\mathrm{PS}_{1}, \mathrm{PS}_{2}, \mathrm{PS}_{3}, \mathrm{PS}_{4}, \mathrm{PS}_{5}, \mathrm{PS}_{6}$, respectivamente.

As estimativas de correlações genéticas entre as características $g$ e $g^{\prime}\left(g \neq g^{\prime}\right)$ foram calculadas como:

$$
r \hat{g}_{\left(g g^{\prime}\right)}=\frac{\hat{\sigma}_{g g^{\prime}}\left(t^{\prime} t\right)}{\sqrt{\hat{\sigma}_{g g}\left(t^{\prime} t\right)^{*} \hat{\sigma}_{g^{\prime} g^{\prime}}\left(t^{\prime} t\right)}}, \text { em que }
$$

$t^{\prime}=t$ se as observações consideradas são de um mesmo período da lactação, e $t^{\prime} \neq t$ se as observações são de períodos diferentes.

\section{RESULTADOS E DISCUSSÃO}

No ajustamento da função Ali e Schaeffer houve dificuldades para atingir o critério de convergência, provavelmente pelo número de parâmetros estimados, conforme já observado por Jamrozik et al. (1997). Portanto, para obtenção da solução do sistema, utilizou-se convergência com estimativas menores que $10^{-9}$. Esse problema não ocorreu quando foi ajustado, o polinômio de Legendre.

Na Tab. 1, apresentam-se o número de parâmetros estimados para cada função e a qualidade do ajustamento em cada análise. A função paramétrica Ali e Schaeffer apresentou o melhor ajuste conforme o critério de informação de Akaike (AIC).

As variâncias residuais foram consideradas constantes durante todo o período considerado, com valores de $0,7199 \mathrm{~kg}^{2}$ para a função Ali e Schaeffer, e de $0,7315 \mathrm{~kg}^{2}$ para o polinômio de Legendre.

As matrizes com os componentes de (co)variância dos coeficientes de regressão aleatórios têm dimensões iguais a 5x5 para Ali e Schaeffer e 4x4 para o polinômio de Legendre. Os componentes de (co)variância atribuídos aos coeficientes de regressão ou cada efeito aleatório para cada função constam das Tab. 2 e 3. 
Tabela 1. Número de parâmetros estimados (NP), valor de -2log da função de verossimilhança (-2log L) e critério de informação Akaike (AIC) para os diferentes modelos de regressão aleatória

\begin{tabular}{lccc} 
Modelo & NP & $-2 \log \mathrm{L}$ & AIC \\
\hline Ali e Schaeffer & 31 & 24042.0633 & 24104.0633 \\
Legendre 4 & 21 & 24558.1689 & 24600.1689 \\
\hline
\end{tabular}

Tabela 2. Componentes de (co)variância (diagonal e acima da diagonal), correlações (abaixo da diagonal) atribuídos aos coeficientes de regressão, variâncias residuais e autovalores das matrizes de covariâncias dos efeitos genético aditivo direto e permanente de ambiente estimados de acordo com a função de Ali e Schaeffer

\begin{tabular}{|c|c|c|c|c|c|c|c|c|c|c|}
\hline & \multicolumn{5}{|c|}{ Genético aditivo direto } & \multicolumn{5}{|c|}{ Permanente de ambiente } \\
\hline & $\mathrm{a}_{0}$ & $\mathrm{a}_{1}$ & $\mathrm{a}_{2}$ & $\mathrm{a}_{3}$ & $\mathrm{a}_{4}$ & $\mathrm{a}_{0}$ & $\mathrm{a}_{1}$ & $\mathrm{a}_{2}$ & $\mathrm{a}_{3}$ & $\mathrm{a}_{4}$ \\
\hline $\mathrm{a}_{0}$ & 5,552 & $-2,157$ & $-5,172$ & $-3,297$ & 0,393 & 12,74 & $-0,046$ & $-11,36$ & $-9,699$ & 1,926 \\
\hline $\mathrm{a}_{1}$ & $-0,7926$ & 1,335 & 1,854 & 0,9843 & $-0,1031$ & $-0,7396$ & 0,0003 & 0,0438 & 0,0373 & 0,0074 \\
\hline $\mathrm{a}_{2}$ & $-0,9287$ & 0,6788 & 5,587 & 3,979 & $-0,5608$ & $-0,9195$ & 0,7261 & 11,98 & 9,137 & $-1,788$ \\
\hline$a_{3}$ & $-0,77$ & 0,4688 & 0,9262 & 3,303 & $-0,5149$ & $-0,8837$ & 0,6962 & 0,8583 & 9,455 & $-1,983$ \\
\hline $\mathrm{a}_{4}$ & 0,5478 & $-0,2932$ & $-0,7793$ & $-0,9307$ & 9,27E-02 & 0,821 & $-0,6473$ & $-0,7861$ & $-0,9811$ & 0,4319 \\
\hline Autovalor & 5,22E-03 & $8,76 \mathrm{E}-02$ & 0,306 & 1,352 & 14,12 & 0,0001 & 0,0098 & 0,9529 & 1,601 & 32,05 \\
\hline Residual & & & & & 0,719 & & & & & \\
\hline
\end{tabular}

Os autovalores das matrizes de covariância dos coeficientes de regressão dos efeitos aleatórios constam das Tab. 2 e 3. Há um autovalor muito pequeno em relação ao maior para cada efeito aleatório nos dois modelos. Isto indica que tanto a equação cúbica e a logarítmica se ajustaram de forma eficaz aos efeitos analisados (Nobre et al., 2003).

As herdabilidades para as seis medidas de persistência foram estimadas a partir das matrizes de (co)variância genética aditiva e de ambiente permanente dos coeficientes de regressão aleatória. Estas estimativas estão apresentadas na Tab. 4.

Tabela 3. Componentes de (co)variância (diagonal e acima da diagonal), correlações (abaixo da diagonal) atribuídos aos coeficientes de regressão, variâncias residuais e autovalores das matrizes de covariâncias dos efeitos genético aditivo direto e permanente de ambiente estimados de acordo com o polinômio de Legendre

\begin{tabular}{|c|c|c|c|c|c|c|c|c|}
\hline & \multicolumn{4}{|c|}{ Genético aditivo direto } & \multicolumn{4}{|c|}{ Permanente de ambiente } \\
\hline & $\mathrm{a}_{0}$ & $\mathrm{a}_{1}$ & $\mathrm{a}_{2}$ & $\mathrm{a}_{3}$ & $\mathrm{a}_{0}$ & $\mathrm{a}_{1}$ & $\mathrm{a}_{2}$ & $\mathrm{a}_{3}$ \\
\hline$a_{0}$ & 1,715 & $-0,398$ & 2,63E-02 & 3,81E-02 & 3,387 & $-0,1196$ & $-0,3523$ & $-3,77 \mathrm{E}-02$ \\
\hline$a_{1}$ & $-0,5099$ & 0,3553 & $-6,67 \mathrm{E}-03$ & $-2,36 E-02$ & $-8,78 \mathrm{E}-02$ & 0,5482 & $-3,50 \mathrm{E}-02$ & $-6,14 \mathrm{E}-02$ \\
\hline$a_{2}$ & 4,42E-02 & $-2,47 \mathrm{E}-02$ & 0,2057 & $-6,77 \mathrm{E}-02$ & $-0,3806$ & $-9,40 \mathrm{E}-02$ & 0,253 & 1,25E-02 \\
\hline$a_{3}$ & 0,1833 & $-0,2493$ & $-0,9403$ & 2,52E-02 & $-6,52 \mathrm{E}-02$ & $-0,2645$ & 7,90E-02 & 9,84E-02 \\
\hline Autovalor & $7,14 \mathrm{E}-04$ & 0,2272 & 0,2489 & 1,824 & 8,93E-02 & 0,2071 & 0,5592 & 3,431 \\
\hline Residual & \multicolumn{8}{|c|}{0,7315} \\
\hline
\end{tabular}

Os valores estimados de herdabilidade para cada uma das medidas de persistência variaram de 0,20 a 0,42 para a função Ali e Schaeffer, e de 0,20 a 0,39 para o polinômio de Legendre. Estas estimativas são maiores que as encontradas por Cobuci et al. (2004), que obtiveram valor máximo para estas mesmas medidas, em vacas da raça Holandesa, de 0,27. Isto se justifica pelo fato de que os parâmetros genéticos são específicos para raça, ou mesmo para a amostra utilizada na análise. 
Tabela 4. Estimativas de herdabilidades para as seis medidas de persistência na lactação, para cada função

\begin{tabular}{lcc} 
& Ali e Schaeffer & $\begin{array}{c}\text { Polinômio de } \\
\text { Legendre }\end{array}$ \\
\hline $\mathrm{PS}_{1}$ & 0,30 & 0,28 \\
$\mathrm{PS}_{2}$ & 0,39 & 0,38 \\
$\mathrm{PS}_{3}$ & 0,42 & 0,39 \\
$\mathrm{PS}_{4}$ & 0,20 & 0,20 \\
$\mathrm{PS}_{5}$ & 0,32 & 0,25 \\
$\mathrm{PS}_{6}$ & 0,36 & 0,29 \\
\hline
\end{tabular}

$\mathrm{PS}_{1}$ a $\mathrm{PS}_{6}$ indicam as medidas de persistência

A parte da curva de lactação utilizada para calcular a persistência pode resultar em maior ou menor estimativa de herdabilidade. Segundo Swalve (1995), as herdabilidades para produção de leite são maiores no período intermediário da lactação, portanto medidas de persistência que utilizam apenas este período podem ter maior herdabilidade do que uma medida que considera todos os períodos da curva.

As correlações genéticas e de ambiente entre as medidas de persistência constam da Tab. 5, e são muito semelhantes para as duas funções estudadas. Além disso, correlações genéticas e de ambiente mantiveram as mesmas tendências e magnitudes. Os valores apresentam grande variação, sendo observados sinais negativos para correlações entre a medida $\mathrm{PS}_{5}$ e as demais, em razão de essa medida representar o desvio dos valores genéticos acumulados em relação ao valor genético aos 280 dias, ou seja, contraria as demais que medem o desvio da produção adicional da fase inicial da lactação.

Tabela 5. Correlações genéticas e de ambiente para a função Ali e Schaeffer (acima da diagonal) e para o polinômio de Legendre (abaixo da diagonal) entre as medidas de persistência na lactação

\begin{tabular}{|c|c|c|c|c|c|c|c|c|c|c|c|c|}
\hline & \multicolumn{6}{|c|}{ Correlação genética } & \multicolumn{6}{|c|}{ Correlação ambiental } \\
\hline & $\mathrm{PS}_{1}$ & $\mathrm{PS}_{2}$ & $\mathrm{PS}_{3}$ & $\mathrm{PS}_{4}$ & $\mathrm{PS}_{5}$ & $\mathrm{PS}_{6}$ & $\mathrm{PS}_{1}$ & $\mathrm{PS}_{2}$ & $\mathrm{PS}_{3}$ & $\mathrm{PS}_{4}$ & $\mathrm{PS}_{5}$ & $\mathrm{PS}_{6}$ \\
\hline $\mathrm{PS}_{1}$ & & 0,45 & 0,96 & 0,82 & $-0,91$ & 0,96 & & 0,63 & 0,97 & 0,85 & $-0,85$ & 0,95 \\
\hline $\mathrm{PS}_{2}$ & 0,45 & & 0,68 & 0,87 & $-0,04$ & 0,18 & 0,53 & & 0,78 & 0,93 & $-0,14$ & 0,35 \\
\hline $\mathrm{PS}_{3}$ & 0,95 & 0,71 & & 0,94 & $-0,75$ & 0,84 & 0,94 & 0,78 & & 0,92 & $-0,73$ & 0,86 \\
\hline $\mathrm{PS}_{4}$ & 0,81 & 0,88 & 0,96 & & $-0,50$ & 0,62 & 0,76 & 0,93 & 0,93 & & $-0,44$ & 0,63 \\
\hline $\mathrm{PS}_{5}$ & $-0,93$ & $-0,08$ & $-0,75$ & $-0,53$ & & $-0,99$ & $-0,88$ & $-0,09$ & $-0,68$ & $-0,36$ & & $-0,97$ \\
\hline $\mathrm{PS}_{6}$ & 0,97 & 0,23 & 0,85 & 0,66 & $-0,99$ & & 0,97 & 0,32 & 0,84 & 0,59 & $-0,97$ & \\
\hline
\end{tabular}

$\mathrm{PS}_{1}$ a $\mathrm{PS}_{6}$ indicam as medidas de persistência

Jakobsen et al. (2002), em estudo comparativo entre medidas de persistência na lactação, obtiveram correlações genéticas que variaram de $-0,41$ a 0,93 para as medidas de 1 a 5 , que também foram utilizadas neste estudo. Portanto, os autores obtiveram resultados com maiores valores do que os obtidos neste estudo. Cobuci et al. (2004), ao compararem as seis medidas avaliadas, encontraram variações com magnitude maiores do que 0,83, tanto para correlações genéticas como para as de ambiente e, portanto, também maiores do que as deste estudo.

As correlações genéticas entre as medidas de persistência e as produções de leite em períodos determinados estão apresentadas na Tab. 6.

Tabela 6. Correlação genética entre as medidas de persistência na lactação e produção de leite nos dias determinados da lactação, para a função Ali e Schaeffer e para o polinômio de Legendre

\begin{tabular}{lcccccccccc}
\hline & \multicolumn{1}{c}{ Ali e Schaeffer } & \multicolumn{1}{c}{ Polinômio de Legendre } \\
\cline { 2 - 11 }$y$ & 6 & 30 & 150 & 240 & 305 & 6 & 30 & 150 & 240 & 305 \\
\hline $\mathrm{PS}_{1}$ & $-0,31$ & $-0,57$ & $-0,56$ & 0,05 & 0,48 & $-0,40$ & $-0,58$ & $-0,59$ & 0,02 & 0,34 \\
$\mathrm{PS}_{2}$ & $-0,89$ & $-0,78$ & $-0,15$ & $-0,11$ & $-0,23$ & $-0,90$ & $-0,80$ & $-0,14$ & $-0,14$ & $-0,20$ \\
$\mathrm{PS}_{3}$ & $-0,55$ & $-0,70$ & $-0,47$ & 0,05 & 0,35 & $-0,64$ & $-0,74$ & $-0,51$ & $-0,03$ & 0,19 \\
$\mathrm{PS}_{4}$ & $-0,70$ & $-0,85$ & $-0,46$ & $-0,12$ & 0,05 & $-0,80$ & $-0,83$ & $-0,41$ & $-0,09$ & 0,03 \\
$\mathrm{PS}_{5}$ & $-0,05$ & 0,25 & 0,51 & $-0,17$ & $-0,68$ & 0,07 & 0,31 & 0,59 & $-0,09$ & $-0,47$ \\
$\mathrm{PS}_{6}$ & $-0,07$ & $-0,36$ & $-0,54$ & 0,13 & 0,62 & $-0,21$ & $-0,43$ & $-0,60$ & 0,06 & 0,42 \\
\hline
\end{tabular}

$\mathrm{PS}_{1}$ a $\mathrm{PS}_{6}$ indicam as medidas de persistência 
Observou-se que as correlações encontradas entre as medidas de persistência e a produção de leite aos 305 dias são baixas para as duas funções estudadas. As medidas $\mathrm{PS}_{2}$ e $\mathrm{PS}_{5}$ correlacionaram-se negativamente com a produção aos 305 dias, e a medida $\mathrm{PS}_{4}$ apresentou correlação bem próxima de zero com a produção de leite aos 305 dias. Estas correlações foram menores do que as encontradas por Jakobsen et al. (2002). As correlações entre $\mathrm{PS}_{1}$ e $\mathrm{PS}_{3}$ e produção de leite aos 305 dias, obtidas neste estudo, foram maiores que as observadas pelos mesmos autores. Cobuci et al. (2004), ao estudarem essas medidas em vacas da raça Holandesa, verificaram valores semelhantes aos obtidos neste estudo, excetuando a $\mathrm{PS}_{2}$, que, para os autores, apresentou correlação positiva com a produção aos 305 dias.

Jakobsen et al. (2002), ao encontrarem valor de zero para correlação entre a medida $\mathrm{PS}_{5}$ e a produção aos 305 dias, sugeriram que esta seria a melhor forma de avaliar a persistência. Assim, tem-se que menores valores para as medidas $\mathrm{PS}_{1}$, $\mathrm{PS}_{2}, \mathrm{PS}_{3}, \mathrm{PS}_{4}$ e $\mathrm{PS}_{6}$ indicam uma curva de produção de leite mais persistente, e, para a medida $\mathrm{PS}_{5}$, maior valor indica maior persistência. Portanto, o resultado negativo da correlação entre $\mathrm{PS}_{2}$ e a produção aos 305 dias sugere que os menores valores para persistência, que seria o desejável, estariam associados a maiores produções de leite ao final da lactação.
As correlações entre as medidas de persistência e a produção de leite em períodos determinados da lactação (6, 30, 150 e 240 dias), em geral, apresentaram sinais negativos, com decréscimo da magnitude à medida que a produção de leite se aproxima do período final da lactação. Isso indica que aumentos na produção inicial de leite resultam em diminuição na produção de leite na fase final, ou seja, maiores produções na fase inicial e no pico da lactação poderiam levar à queda na produção final. Segundo Madsen (1975), vacas com alta produção até o pico apresentam taxa acentuada de queda na produção de leite, comparadas às que apresentam menores produções nesta mesma fase.

Na Tab. 7, constam as estimativas de correlação entre ambiente permanente e produção de leite em períodos determinados. Os valores encontrados variaram entre baixo e moderado. Estes resultados assemelham-se aos encontrados por Cobuci et al. (2004) que observaram variações nestas correlações, entre -0,61 e 0,61.

Segundo Jakobsen et al. (2002), para que a persistência na lactação seja incluída nas avaliações genéticas, há necessidade de primeiro determinar o valor econômico desta característica, para estabelecer o peso que deve ser dado a ela em um programa de seleção. Contudo, Dekkers et al. (1996) afirmaram que animais mais persistentes reduzem custos com alimentação, reprodução e saúde, além de aumentarem a produção total de leite.

Tabela 7. Correlações de ambiente entre as medidas de persistência na lactação e a produção de leite nos dias determinados da lactação, para a função Ali e Schaeffer e para o polinômio de Legendre

\begin{tabular}{|c|c|c|c|c|c|c|c|c|c|c|}
\hline & \multicolumn{5}{|c|}{ Ali e Schaeffer } & \multicolumn{5}{|c|}{ Polinômio de Legendre } \\
\hline & 6 & 30 & 150 & 240 & 305 & 6 & 30 & 150 & 240 & 305 \\
\hline $\mathrm{PS}_{1}$ & 0,10 & $-0,60$ & $-0,18$ & 0,22 & 0,50 & $-0,48$ & $-0,57$ & $-0,27$ & 0,18 & 0,44 \\
\hline $\mathrm{PS}_{2}$ & 0,14 & $-0,48$ & 0,32 & 0,47 & 0,27 & $-0,51$ & $-0,42$ & 0,31 & 0,50 & 0,15 \\
\hline $\mathrm{PS}_{3}$ & 0,05 & $-0,61$ & $-0,07$ & 0,30 & 0,48 & $-0,54$ & $-0,58$ & $-0,08$ & 0,34 & 0,37 \\
\hline $\mathrm{PS}_{4}$ & 0,25 & $-0,57$ & 0,14 & 0,41 & 0,38 & $-0,48$ & $-0,49$ & 0,14 & 0,47 & 0,21 \\
\hline $\mathrm{PS}_{5}$ & 0,09 & 0,45 & 0,45 & 0,03 & $-0,47$ & 0,33 & 0,46 & 0,49 & 0,08 & $-0,47$ \\
\hline $\mathrm{PS}_{6}$ & 0,00 & $-0,53$ & $-0,35$ & 0,08 & 0,50 & $-0,40$ & $-0,52$ & $-0,39$ & 0,07 & 0,45 \\
\hline
\end{tabular}

$\mathrm{PS}_{1}$ a $\mathrm{PS}_{6}$ indicam as medidas de persistência

\section{CONCLUSÕES}

A função que melhor se adequou às análises foi a Ali e Schaeffer, porém apresenta problemas de convergência quando se aumenta o número de dados analisados. A persistência na lactação é medida que apresenta moderada herdabilidade e baixa correlação com a produção de leite aos 305 dias; e pode ser usada na seleção para modificar o formato da curva de lactação sem grandes alterações na produção total de leite. Entre as medidas de persistência na lactação em bovinos da raça Guzerá, avaliados neste estudo, a que considera a diferença entre as áreas intermediária 
e inicial sob a curva de lactação $\left(\mathrm{PS}_{2}\right)$ é a que mais se adequou à seleção de animais mais persistentes, resultando, contudo, em baixo ganho genético na produção total de leite.

\section{REFERÊNCIAS BIBLIOGRÁFICAS}

ALI, T.E.; SCHAEFFER, L.R. Accounting for covariances among test day milk yields in dairy cows. Can. J. Anim. Sci., v.67, p.637-644, 1987.

COBUCI, J.A.; EUCLYDES, R.F.; COSTA, C.N. et al. Análises da persistência na lactação de vacas da raça Holandesa, usando produção no dia do controle e modelo de regressão aleatória. Rev. Bras. Zootec., v.33, p.546-554, 2004.

COBUCI, J.A.; COSTA, C.N.; TEIXEIRA, N.M. et al. Utilização dos polinômios de Legendre e da função de Wilmink em avaliações genéticas para persistência na lactação de animais da raça Holandesa. Arq. Bras. Med. Vet. Zootec., v.58, p.614-623, 2006.

DEKKERS, J.C.M.; JAMROZIK, J.; TEM HAG, J.H. et al. Genetic and economic evaluation of persistency in dairy cattle. Proc. Int. Workshop of genetic improvement of functional taits in cattle. Interbull Bull., v.12, p.97-102, 1996.

JAKOBSEN, J.H.; MADESEN, P.; JENSEN, J. et al. Genetic parameters for milk production and persistency for Danish Holstein estimated in random regression models using REML. J. Dairy Sci., v.85, p.1606-1616, 2002.

JAMROZIK, J.; SCHAEFFER, L.R.; DEKKERS, J.C.M. Genetic evaluation of dairy cattle using teste day yields and random regression model. J. Dairy Sci., v.80, p.12171226, 1997.

JAMROZIK, J.; SCHAEFFER, L.R. Estimates of genetic parameters for a test day model with random regression for yield traits of first lactation Holstein. J. Dairy Sci., v.80, p.762-770, 1997.
JENSEN, J. Genetic evaluation of dairy cattle using test-day models. J. Dairy Sci., v.84, p.2803-2812, 2001.

KIRKPATRICK, M.; LOFSVOLD, D.; BULMER, M. Analysis of the inheritance, selection of growth trajectories. Genetics, v.124, p.979-993, 1990.

MADSEN, O. A comparison of some suggested measures of persistency of milk yield in dairy cows. Anim. Prod., v.20, p.191-197, 1975.

MEYER, K.; HILL, W.G. Estimation of genetic and phenotypic covariance functions for longitudinal or repeated records by restricted maximum likelihood. Livest. Prod. Sci., v.47, p.185-200, 1997.

NOBRE, P.R.C.; MISZTAL, I.; TSURUTA, S. et al. Analyses of growth curves of Nelore attle by multiple-trait and random regression models. J. Anim. Sci., v.81, p.918-926, 2003.

SCHAEFFER, L.R. 2001. Randon regression. http://www.aps.uoguelph.ca/ Irs/ANSC637/LRS 14/ Acessado em: 12 set. 2007.

SCHAEFFER, L.R. Application of random regression models in animal breeding. Liv. Prod. Sci., v.86, p.35-45, 2004.

SWALVE, H.H. Test day models in the analysis of dairy production data - a review. Arch. Tierz., Dummerstorf, v.38, p.591-612, 1995.

WINKLER, R.; PENNA, V.M. O Guzerá. Inf. Agropec., v.16, p.10-14, 1992.

WOOD, P.D.P. Algebric model of lactation curve in cattle. Nature, v.216, p.164-165, 1967. 